\title{
Thermal Radiation from Nucleons and Mesons
}

\author{
Jan-e Alam ${ }^{1}$, Pradip Roy ${ }^{2}$ and Sourav Sarkar ${ }^{1}$ \\ ${ }^{1}$ Variable Energy Cyclotron Centre, Kolkata 700064, India \\ ${ }^{2}$ Saha Institute of Nuclear Physics, Kolkata 700064, India
}

(Dated: February 5, 2020)

\begin{abstract}
Thermal photon emission rates due to meson-nucleon interactions have been evaluated. An exhaustive set of reactions involving $p(\bar{p}), n(\bar{n}), \rho, \omega, a_{1}, \pi$ and $\eta$ is seen to provide a sizeable contribution to the emission rate from hot hadronic matter. Contributions from baryonic resonances are found to be negligibly small.
\end{abstract}

PACS numbers: 25.75.-q,12.40.Vv,13.85.Qk,21.65.+f

With results coming in from $\mathrm{Au}-\mathrm{Au}$ collisions at the Relativistic Heavy Ion Collider (RHIC) at Brookhaven National Laboratory (BNL), the search for signals of quark gluon plasma (QGP) is being pursued with renewed vigour. It is well known that electromagnetic signals are the only ones which probe the entire space-time history of the collisions as they are emitted at all stages and undergo very little re-scattering. Since signals from QGP have to be unearthed from the huge debris of radiations from hadronic sources it becomes all the more necessary to have an accurate estimation of the same. Apart from this the emission of photons from the hadronic matter is also important to investigate the modifications of the hadronic spectral functions in a thermal bath [1].

The hadronic matter is usually considered to be a gas of the low lying mesons $\pi, \rho, \omega$ and $\eta$. Reactions between these as well as the decays of the $\rho$ amd $\omega$ were considered 2 to be the source of thermal photons from hadronic matter. In [3] the role of the intermediary $a_{1}$ meson in the $\pi \rho \rightarrow \pi \gamma$ channel was discussed. We [4] found an enhancement in the photon production by incorporating medium effects through thermal loop corrections on the hadronic decay widths and masses. In our quest for additional sources of thermal photon production we failed to find discussions on the role of baryons and a priori we find no reason for this. We hence consider it worthwhile to investigate, in particular, the role of meson-nucleon interactions for photon emission. In an earlier work [5] we had found the contribution due to the decay of baryon resonances to be small.

In this work we evaluate photon emission due to nucleon (and antinucleon) scattering from $\pi, \rho, \omega$, $\eta$ and $a_{1}$ mesons in the thermal bath. Listed below are the standard phenomenological interactions which have been used:

$$
\begin{aligned}
\mathcal{L}_{V N N} & =g_{\rho N N}\left[\bar{N} \gamma^{\mu} \boldsymbol{\tau} N \cdot \boldsymbol{\rho}_{\mu}-\frac{\kappa}{2 m_{N}} \bar{N} \sigma^{\mu \nu} \boldsymbol{\tau} N \cdot \partial_{\nu} \boldsymbol{\rho}_{\mu}\right]-g_{\omega N N} \bar{N} \gamma^{\mu} N \omega_{\mu} \\
\mathcal{L}_{A N N} & =\frac{g_{\pi N N}}{m_{\pi}} m_{a_{1}} \bar{N} \gamma^{5} \gamma^{\mu} \boldsymbol{\tau} N \cdot \boldsymbol{a}_{\mathbf{1}_{\mu}} \\
\mathcal{L}_{P N N} & =\frac{g_{\pi N N}}{m_{\pi}} \bar{N} \gamma^{5} \gamma^{\mu} \boldsymbol{\tau} N \cdot \partial_{\mu} \boldsymbol{\pi}+\frac{g_{\eta N N}}{m_{\eta}} \bar{N} \gamma^{5} \gamma^{\mu} N \partial_{\mu} \eta \\
\mathcal{L}_{e m} & =e A^{\mu}\left[\bar{N} \gamma_{\mu} N-\left[\boldsymbol{\pi} \times \partial_{\mu} \boldsymbol{\pi}-\boldsymbol{\rho}^{\nu} \times\left(\partial_{\nu} \boldsymbol{\rho}_{\mu}-\partial_{\mu} \boldsymbol{\rho}_{\nu}\right)\right]_{3}\right]+\frac{e}{2} F^{\mu \nu}\left(\boldsymbol{\rho}_{\mu} \times \boldsymbol{\rho}_{\nu}\right)_{3}+g_{a_{1} \pi \gamma} \boldsymbol{a}_{1 \mu} \cdot \partial_{\nu} \boldsymbol{\pi} F^{\mu \nu}
\end{aligned}
$$

Here, $N$ represents the nucleon isospin doublet $\left(\begin{array}{c}p \\ n\end{array}\right), F_{\mu \nu}=\partial_{\mu} A_{\nu}-\partial_{\nu} A_{\mu}$ and $\boldsymbol{\tau}$ are the Pauli matrices. We have included monopole form factors at the strong vertices. They have the generic form $\left(\Lambda^{2}-M^{2}\right) /\left(\Lambda^{2}-X^{2}\right)$ where $\Lambda$ and $M$ stand for the cutoff and the mass of the exchanged particle in the $X(=t / u)$ channels respectively. The values of the cutoff and the coupling constants have been taken from Ref. 6] and are listed in Table 1. It is to be noted that the number of Feynman diagrams involved in the present calculations with all the isospin combinations of mesons and baryons is very large. The invariant amplitudes for these diagrams will require too large a space to be presented here.

In Fig. 1 we plot the rate of photon emission from a thermal hadronic medium consisting of mesons and baryons at $T=170 \mathrm{MeV}$ and zero baryonic chemi- 


\begin{tabular}{cccc}
\hline \hline Vertex & Process & Coupling & $\Lambda(\mathrm{GeV})$ \\
\hline$N N \pi$ & $\pi$ exchange & $\frac{g_{\pi N N}^{2}}{4 \pi}=0.079$ & 0.6 \\
& $N$ exchange & 1.3 \\
$N N \eta$ & $N$ exchange & $\frac{g_{\eta N N}^{2}}{4 \pi}=0.148$ & 2.5 \\
$N N \rho$ & $\rho$ exchange & $\frac{g_{\rho N N}^{2}}{4 \pi}=0.84$ & 1.4 \\
& & $\kappa=6.1$ & \\
& $N$ exchange & 1.2 \\
$N N \omega$ & $N$ exchange & $\frac{g_{\omega N N}^{2}}{4 \pi}=20.0$ & 1.2 \\
$a_{1} \pi \gamma$ & & $g_{a_{1} \pi \gamma}=0.743$ & \\
\hline
\end{tabular}

TABLE I: Values of the coupling constants and cutoff parameters used.

cal potential. For convenience we have subdivided the large number of photon producing reactions into three categories involving nucleons and pseudoscalar mesons $(P=\pi, \eta)$, vector mesons $(V=\rho, \omega$ and $\phi)$ and axial vector $\left(A=a_{1}\right)$ meson. Type $P$ consists of reactions of the kind $P N \rightarrow \gamma N$ and $N \bar{N} \rightarrow \gamma P$, $V$ of the type $V N \rightarrow \gamma N$ and $N \bar{N} \rightarrow \gamma V$ and $A$ of the type $A N \rightarrow \gamma N$ and $N \bar{N} \rightarrow \gamma A$. Due to the the relatively high abundance of pions in the system, type $P$ has the highest contribution at lower energies. $V$ type reactions are found to dominate after about $1.5 \mathrm{GeV}$. The important factor in this case is the large spin-isospin degeneracy of the $\rho$ meson. Also shown is the contribution to the photon emission rate from the decay of heavy baryons $R$ to nucleons, $N$ and photons, $R \rightarrow \gamma N$. Here $R$ consists of the baryonic resonances $\mathrm{N}(1440), \mathrm{N}(1520)$, $\mathrm{N}(1535), \quad \mathrm{N}(1650), \mathrm{N}(1675), \mathrm{N}(1680), \mathrm{N}(1700)$, $\Delta(1232), \Delta(1620), \Delta(1700), \Delta(1905)$ and $\Delta(1950)$. The partial decay widths of $R$ have been taken from Ref. 7].

In fig. 2 the emission rates from the baryonic degrees of freedom have been shown at a temperature $140 \mathrm{MeV}$. The introduction of the monopole form factors at the meson nucleon vertices reduces the the production rate by a factor of 3 around $E_{\gamma} \sim 2 \mathrm{GeV}$. A similar kind of suppression (see first reference of 2]) has been observed with the inclusion of form factors at the interaction vertices in the emissivity from the reactions involving only mesons $(\pi, \rho, \omega \eta$ and $a_{1}$ ). A factor of $\sim 3$ suppression of the production rate of photons from a mesonic gas will make it comparable with that from the $P, V$ and $A$ types of reactions defined before.

The emission rates of photons from $P, V$ and $A$ types of reactions will become important with increase in temperature. In fact, the production rates

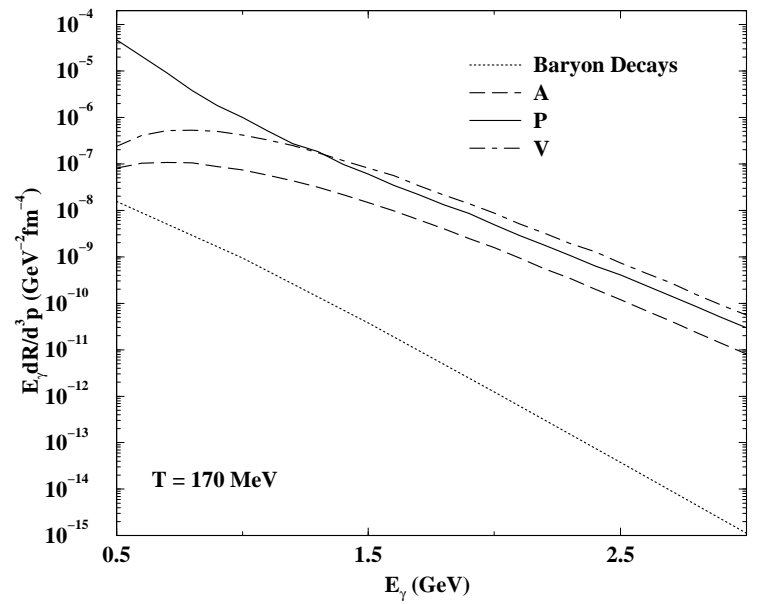

FIG. 1: Thermal photon emission rates from mesonnucleon interactions at $T=170 \mathrm{MeV}$. The legends $P, V$ and $A$ are described in the text.

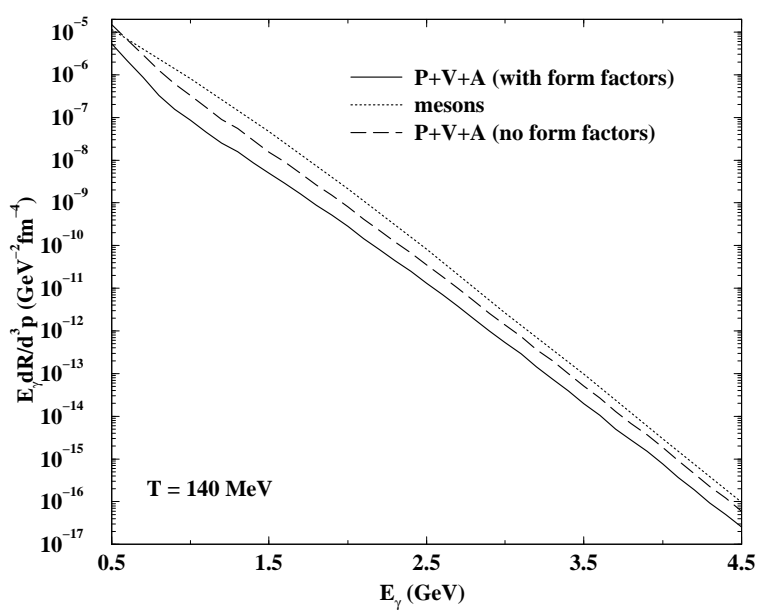

FIG. 2: Thermal photon emission rates at $T=140 \mathrm{MeV}$. Solid and dashed line indicate emission rate from a hadronic gas involving nucleons with and without form factors respectively. The parameters of the form factors are given in Table I. The dotted line represents result from a mesonic gas of $\pi, \rho, \omega \eta$ and $a_{1}$.

with only mesonic degrees of freedom is comparable to the corresponding rates from $P, V$ and $A$ type of reactions if the effects of the vertex form factors are ignored (fig. 31). Considering the fact that the form factors at the interaction vertices suppress the emission rates from mesons as well as from reactions involving nucleons, the production rates involving nucleons are not negligible.

In summary, we have evaluated the production rate of photons from an exhaustive set of reactions involving mesons and baryons. In all the earlier works, the role of the baryons on the photon emis- 


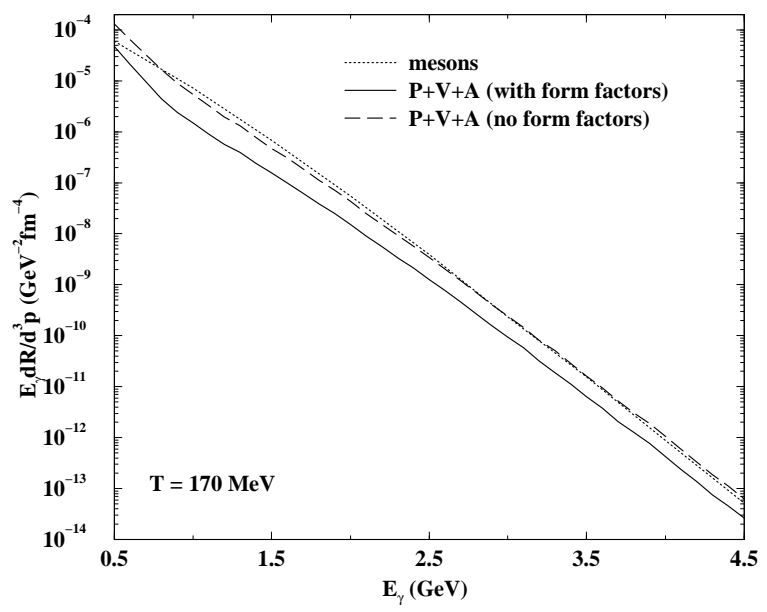

FIG. 3: Same as Fig. 2 at $T=170 \mathrm{MeV}$. sion rates were neglected, though it appears to be important. We observe that the production rates from the reactions involving nucleons are suppressed primarily because of the inclusion of the form factors at the meson-nucleon vertices. At non-zero baryonic chemical potential the emission rates from the reactions involving nucleonic degrees of freedom may be enhanced. However, for very high colliding energies such as at RHIC the value of the chemical potential is expected to be small.
[1] J. Alam, S. Sarkar, P. Roy, T. Hatsuda and B. Sinha, Ann. Phys. (NY), 286, 159 (2000).

[2] J. I. Kapusta, P. Lichard, and D. Seibert, Phys. Rev. D 44, 2774 (1991); C. Song, Phys. Rev. C 47, 2861 (1993).

[3] L. Xiong, E. V. Shuryak, and G.E. Brown, Phys. Rev. D 46, 3798 (1992); J. K. Kim, P. Ko, K. Y. Lee, and S. Rudaz, Phys. Rev. D 53, 4787 (1996).

[4] S. Sarkar, J. Alam, P. Roy, A. K. Dutt-Mazumder, B. Dutta-Roy and B. Sinha, Nucl. Phys. A 634, 206
(1998); P. Roy, S. Sarkar, J. Alam and B. Sinha, Nucl. Phys. A 653, 277 (1999).

[5] J. Alam, P. Roy, S. Sarkar and B. Sinha, nucl-th/0106038 Phys. Rev. C (in press).

[6] O. Krehl, C. Hanhart, S. Krewald and J. Speth, Phys. Rev. C 62025207 (2000).

[7] R. M. Barnett, et al., Particle Data Group, Phys. Rev. D 54, 1 (1996). 